\title{
A rare cause of agranulocytosis
}

\author{
Helena Luís*, Carolina Barros, Mauro Fernandes and Sofia Granito \\ Department of Internal Medicine, Hospital Central do Funchal - SESARAM, E.P.E., Funchal, Portugal
}

\begin{abstract}
Clozapine, an atypical antipsychotic agent, is useful in the treatment of patients with psychotic symptoms. However, severe adverse effects, such as agranulocytosis, can restrict its indications. We present a case of a 42-year-old Caucasian woman with a 4-year history of persistent delusion disorder, who presented with fatigue and fever and was ultimately diagnosed with agranulocytosis due to clozapine. Clozapine-induced agranulocytosis is an uncommon condition, but potentially fatal in consequences. Each patient with an episode of agranulocytosis should be assessed individually, with special attention to risk factors. Upon that, the decision about clozapine rechallenge or withdrawal should be made.
\end{abstract}

\section{Introduction}

Clozapine, a second-generation antipsychotic, is considered the antipsychotic of choice for patients with schizophrenia or schizoaffective disorder whose symptoms are partially or fully resistant to treatment with other antipsychotic drugs or accompanied by persistent suicidal or self-injurious behaviour [1]. However, its use is limited by the risk of haemotological side effects and the need for regular monitoring of haemogram [2,3]. The most serious side effect of clozapine is agranulocytosis with two phenotypes on a spectrum of severity [4], starting from benign neutropenia (cumulative incidence $2,7 \%$ during the first year of use) with absolute neutrophil count (ANC) $\leq 1,5 \times 10^{3} / \mu \mathrm{L}$ to possibly fatal agranulocytosis with ANC $\leq 0.5 \times 10^{3} / \mu \mathrm{L}$ (cumulative incidence $0,8 \%$ at 1 year and $0,91 \%$ at 18 months) [1,5]. The risk of clozapine-induced agranulocytosis and neutropenia is highest in the first 6 months and higher in the initial 18 months after the onset of treatment $[1,6]$.

The mechanism of clozapine-induced agranulocytosis has not been fully described. Neither clozapine nor its major stable metabolite, $\mathrm{N}$-desmethylclozapine, at therapeutic drug concentrations have been found to be directly cytotoxic or interfere with the turnover of bone marrow precursor cells [4,7].

Significant and independent factors associated with clozapinerelated agranulocytosis are female sex, older age5, and use of concomitant medication [8].

Management of clozapine-induced agranulocytosis orders immediate discontinuation of the drug1. To prevent morbidity and mortality from infection, antibiotics should be instituted promptly for febrile neutropenia $[1,5]$. Colony-stimulating factor are also often used 1 since their use may reduce the duration of agranulocytosis by 4 to 5 days [5].

\section{Case presentation}

A 42-year-old Caucasian woman presented to the Emergency Department (ED) of our hospital with one-month fatigue. She also had a fever started 3 days before. For a dental abscess, she was receiving amoxicillin/clavulanic acid 875/125 mg, paracetamol $1000 \mathrm{mg}$, and diclofenac $50 \mathrm{mg}$ for the previous 4 days.

There was no history of psychiatric disorders until 12 years ago when the patient had a postpartum depressive episode after the birth of her second daughter. The patient started antidepressive therapy with sertraline and completed it for 3 years, with progressive weaning until suspension. Four years later, she developed persistent delusion disorder (a highly systematic delusion with the consistency of the paranoid theme). The patient had been hospitalized the year before, due to the intensification of the delusional activity dynamism. She started antipsychotic therapy with paliperidone and olanzapine titrated to an effective dose without any repercussions on delusional activity. For that reason, refractory psychosis was assumed, and clozapine was instituted gradually up to $300 \mathrm{mg}$ per day. The patient also started depot therapy with paliperidone palmitate $150 \mathrm{mg}$ per month to ensure greater adherence to the therapeutic regimen. With clozapine titration, significant improvement in delusional ideation and greater affective distance from it was reported in the first months of treatment.

At the ED, the patient was pale, febrile, and her blood pressure was $118 / 67 \mathrm{mmHg}$, while her pulse was 118 beats per minute and regular. Heart and breath sounds were normal. There was no hepatosplenomegaly, lymphadenopathy, abdominal tenderness, or mass. The patient had ulceration of the perianal region with exuberant inflammatory signs suggestive of candidiasis.

The main investigations of the patient showed, on blood samples, agranulocytosis (leukopenia $0,6 \times 10^{3} / \mu \mathrm{L}$ and severe neutropenia $\left.0,0 \times 10^{3} / \mu \mathrm{L}\right)$ and lymphopenia $\left(0,6 \times 10^{3} / \mu \mathrm{L}\right)($ Table 1$)$. The hemoglobin

${ }^{\star}$ Correspondence to: Helena Luís; Department of Internal Medicine, Hospital Central do Funchal - SESARAM, E.P.E., Funchal, Portugal, Avenida Luís de Camões No 57, 9004-514 Funchal, Portugal, E-mail: helena.luis@sesaram.pt

Key words: clozapine, agranulocytosis, neutropenia

Received: February 22, 2021; Accepted: March 01, 2021; Published: March 05, 2021 
level was $12,2 \mathrm{~g} / \mathrm{dL}$ and plaquette count $405 \times 10^{3} / \mu \mathrm{L}$. The serum sodium was $132 \mathrm{mEq} / \mathrm{L}$, serum potassium $3,2 \mathrm{mEq} / \mathrm{L}$, alanine aminotransferase (ALT) 88,2 U/L, aspartate aminotransferase (AST) 38,9 U/L, gammaglutamyltransferase $(\gamma \mathrm{GT}) 132,3 \mathrm{U} / \mathrm{L}$, lactate dehydrogenase $158 \mathrm{U} / \mathrm{L}$ and C-reactive protein (CRP) was $334,96 \mathrm{mg} / \mathrm{L}$. Chest X-ray and ECG were performed and both were normal.

She was diagnosed as having drug-induced agranulocytosis with febrile neutropenia. Clozapine was immediately withdrawal and she was maintained on strict aseptic precautions and isolation procedures. Bone marrow aspiration revealed a dry tap. The patient started therapy with filgrastim, a granulocyte colony-stimulating factor (G-CSF), on day 1. Blood cultures were collected, and the patient started IV empirical antibiotic therapy with piperacillin/tazobactam $4,5 \mathrm{gr}$. She was admitted under the department of Hematology. To the initial regimen, fluconazole $100 \mathrm{mg}$ was added for the management of candidiasis.

During hospitalization, further investigation was performed. Thyroid hormones were within normal limits. Serologic tests, hepatitis $\mathrm{B}$, hepatitis $\mathrm{C}$, anti-DNA, antinuclear, proteinase 3 , antineutrophil cytoplasmic (PR3-ANCA and cANCA), perinuclear antineutrophil cytoplasmic antibodies (pANCA), and antineutrophil cytoplasmic antibodies against myeloperoxidase (MPO-ANCA), were negative. The blood cultures were also negative.

On the third day of hospitalization, the patient maintained paranoid ideation that emerged during the interview, without significant psychotic distress. She had depressive mood and anxiety, configuring an adjustment disorder. Lorazepam 2,5 $\mathrm{mg}$ at bedtime was introduced and then increased to $3,5 \mathrm{mg}$. It was deferred the introduction of any other therapy until analytical improvement.

After the first course of antibiotic therapy, fever persisted. The patient collected two blood cultures and urine culture and started a new antibiotic therapy with meropenem 1 gr. Both cultures were negative.

On the eighth day, the patient was psychopathologically worst, with progression to depressive mood, emotional lability, initial insomnia, and polifragmented sleep with recurrent nighttime awakenings. The past spontaneous delusional activity did not emerge. Regarding insomnia, with a high impact on mood, she started zolpidem $10 \mathrm{mg}$ at bedtime.

Despite antibiotic and antifungal therapy, the patient maintained fever and pain in the perianal region. Rectal examination was impossible to perform due to the patient's intolerance. A perianal abscess was assumed, and the patient started another antibiotic therapy with vancomycin. Magnetic resonance was performed and showed concentric thickening of the low rectum wall, associated with adenopathies in the adjacent perirectal fat, findings suspected of neoplastic lesions (Figure 1). Adjacent to the posterior aspect of the anal sphincter complex, there were imaging changes compatible with an active inflammatory process, but without evidence of fistulous trajectories or suspicious collections. Colonoscopy was performed and showed, in the rectum at $8 \mathrm{~cm}$ from the anal margin, an area of mucosal retraction with a scarred aspect and pseudopolyps (Figure 2). Biopsies were performed and revealed nonspecific morphological changes.

During and after granulocyte treatment, her neutrophil and monocyte counts rose, and her clinical condition improved. Filgrastim was suspended on the twenty-third day and a myelogram was performed, showing granulocytic lineage. Flow cytometry performed revealed $2 \%$ of blast population with normal phenotype. Table 1 demonstrates the course of the patient's white blood count during and after granulocyte treatment.

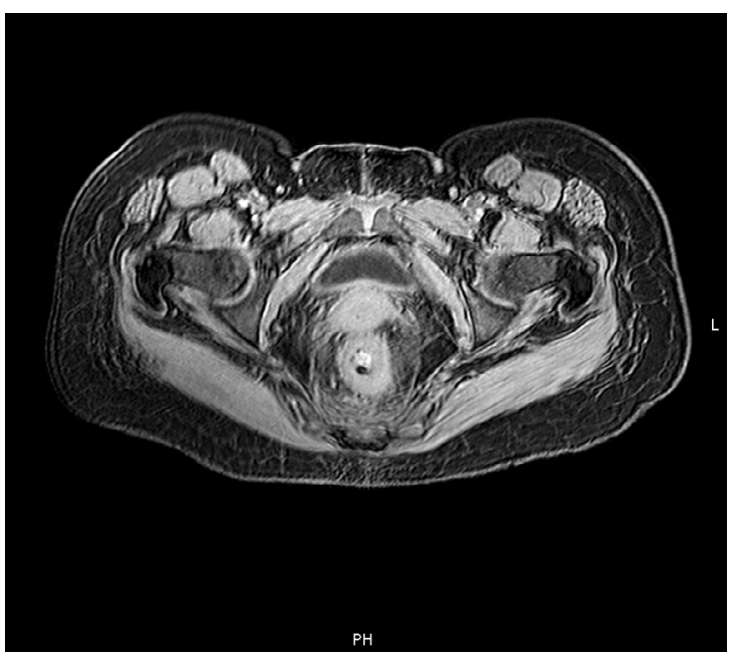

Figure 1. Magnetic resonance showing concentric thickening of the low rectum wall

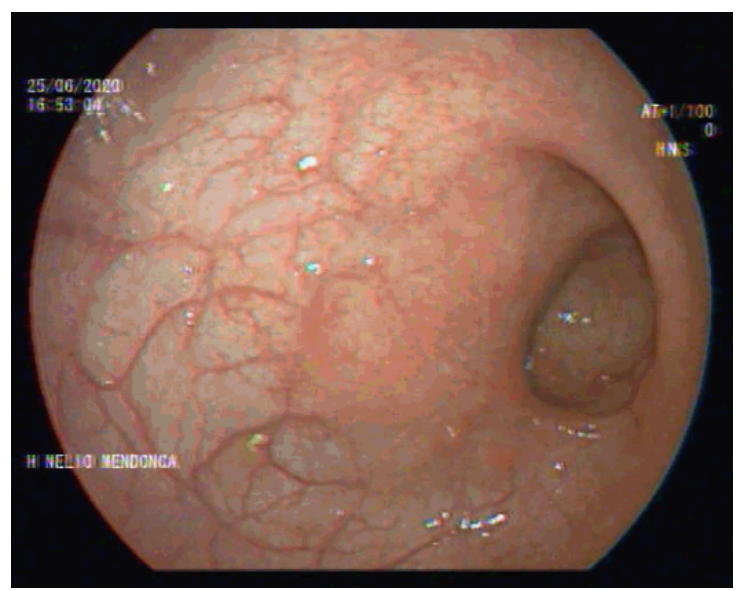

Figure 2. Colonoscopy showing mucosal retraction with a scarred aspect of the rectum

The patient was discharged. She suspended zolpidem $10 \mathrm{mg}$ and initiated flurazepam $15 \mathrm{mg}$, with follow-up by Psychiatry and Hematology. Taking into account the agranulocytosis, as well as the reduction of the dynamism of the delusional activity without functional repercussion, it was decided not to reintroduce any antipsychotic for now.

\section{Discussion}

We presented a case of clozapine-induced neutropenia and agranulocytosis in a patient with persistent delusion disorder treated with clozapine for 3 months.

Clozapine-induced agranulocytosis is a rare condition in comparison with other fatal complications of clozapine treatment (i.e., bowel obstruction, myocarditis) and it needs to be properly detected and addressed [7].

Clozapine can induce two clinically distinct types of agranulocytosis on a spectrum of severity, starting from benign neutropenia (cumulative incidence $2,7 \%$ during the first year of use) with an absolute neutrophil count $\left(\right.$ ANC) $\leq 1,5 \times 10^{3} / \mu \mathrm{L}$ to possibly fatal agranulocytosis with ANC $\leq 0.5 \times 10^{3} / \mu \mathrm{L}$ (cumulative incidence $0,8 \%$ at 1 year and $0,91 \%$ at 18 months) [1,5]. In the first type, when clozapine is discontinued, recovery is usually rapid (2-8 days) [9] but in the second type, as in 
Table 1. The course of the patient's white blood cell count during and after granulocyte treatment

\begin{tabular}{|c|c|c|c|c|c|c|c|}
\hline Laboratory test & Normal range & Day 1 & Day 3 & Day 7 & Day 12 & Day 17 & Day 23 \\
& & & & Day without \\
\hline White blood cell count & $4,5-11,0 \times 10^{3} / \mu \mathrm{L}$ & 0,5 & 0,9 & 1,7 & 1,2 & 1,4 \\
\hline Neutrophils & $2,0-7,5 \times 10^{3} / \mu \mathrm{L}$ & 0,0 & 0,0 & 0,0 & 0,2 & 0,2 \\
\hline Lymphocytes & $1,5-4,0 \times 10^{3} / \mu \mathrm{L}$ & 0,4 & 0,9 & 1,7 & 0,2 & 0,2 \\
\hline Monocytes & $0,0-1,0 \times 10^{3} / \mu \mathrm{L}$ & 0,0 & 0,0 & 0,0 & 0,0 & 0,7 \\
\hline
\end{tabular}

the present case, even if clozapine is discontinued, agranulocytosis generally lasts for 14-21 days [9].

The incidence of agranulocytosis is reversible in most cases once clozapine is withdrawn promptly. In our patient, immediate discontinuation of clozapine upon diagnosis and G-CSF introduction managed the increase in the neutrophil count and the improvement of the patient's clinical presentation.

The pathophysiologic mechanisms that produce clozapine-induced agranulocytosis are not well understood. There is an age-related increase in the risk of $53 \%$ per decade [9]. The risk of neutropenia or agranulocytosis is not, however, dose related [9]. Furthermore, some authors have suggested an increased incidence in women [9].

The role of the physician is to carefully assess modifiable risk factors, focusing on drug-drug interactions and potential interactions associated with clozapine metabolism. The decision for treatment continuation in the case of clozapine-induced agranulocytosis should be made based on careful consideration of risk factors.

\section{References}

1. Patel R, Lima A, Burke C, Hoffman M (2019) Monocytopenia in clozapine-induced agranulocytosis: Insights into pathophysiology and treatment. BMJ Case Rep 12: e226016. [Crossref]
2. Manu P, Lapitskaya Y, Shaikh A, Nielsen J (2018) Clozapine rechallenge after major adverse effects: Clinical guidelines based on 259 cases. Am J Ther 25: e218-e223. [Crossref]

3. Grover S, Balachander S, Chakarabarti S, Avasthi A (2015) Prescription practices and attitude of psychiatrists towards clozapine: A survey of psychiatrics from India. Asian $J$ Psychiatr 18: 57-65. [Crossref]

4. Li K, Gurrera R, Delisi L (2018) Potentially fatal outcomes associated with clozapine. Schizophr Res 199: 386-389. [Crossref]

5. Mijovic A, MacCabe JH (2020) Clozapine-induced agranulocytosis. Anna Hematol 99 2477-2482.

6. Velayudhan R, Kakkan S (2014) Late onset clozapine induced agranulocytosis. Indian $J$ Psychol Med 36: 425-427. [Crossref]

7. Wicinski M, Weclewicz M (2018) Clozapine-induced agranulocytosis/ granulocytopenia: mechanisms and monitoring. Curr Opin Hematol 25: 22-28.

8. Balda V, Garay O, Papale R, Inés B, Viviana GB (2015) Clozapine-associated neutropenia and agranulocytosis in Argentina (2007-2012). Int Clin Psychopharmacol 30: 109-114. [Crossref]

9. Voulgari C, Giannas R, Paterakis G (2015) Clozapine-induced late agranulocytosis and severe neutropenia complicated with streptococcus pneumonia, venous thromboembolism, and allergic vasculitis in treatment-resistant female psychosis. Case Reports Med 2015: 703218

Copyright: (C2021 Luís H. This is an open-access article distributed under the terms of the Creative Commons Attribution License, which permits unrestricted use, distribution, and reproduction in any medium, provided the original author and source are credited. 